\title{
Predictors of poor treatment outcome in multi- and extensively drug-resistant
} pulmonary TB

\author{
K. Kliiman and A. Altraja
}

ABSTRACT: Treatment outcome in multidrug-resistant (MDR) and extensively drug-resistant (XDR) tuberculosis (TB) is often unsuccessful, but the particular determinants of poor treatment outcome have remained obscure. The present authors therefore analysed treatment effectiveness and predictors of poor treatment outcome in pulmonary MDR-TB and XDR-TB in Estonia, a European country with one of the highest MDR-TB and XDR-TB rates in the world.

All culture-confirmed pulmonary MDR-TB and XDR-TB patients who started TB treatment in 2003-2005 were included. Multivariate analysis was performed on two models of predictors: 1) patients' HIV-status, demographic and socioeconomic characteristics; and 2) TB-related data.

In the 235 MDR-TB patients, the proportion of overall successful treatment outcome was $60.4 \%$, rising to $72.8 \%$ among adherent patients. Among the 54 XDR-TB patients, these proportions were $\mathbf{4 2 . 6 \%}$ and $\mathbf{5 0 . 0 \%}$, respectively. Risk factors for poor treatment outcome in MDR-TB were HIV infection, previous TB treatment, resistance to ofloxacin and positive acid-fast bacilli (AFB) smear at the start of treatment. Predictors of poor treatment outcome in XDR-TB were urban residence and positive AFB smear.

This country-wide study provides evidence that to improve treatment outcome in multidrugresistant and extensively drug-resistant tuberculosis, special care should be taken to treat HIVinfected patients and urban residents, as well as to make efforts to diminish re-treatment cases by increasing patient adherence.

KEYWORDS: Drug resistance, extensively drug-resistant tuberculosis, multidrug-resistant tuberculosis, treatment outcome, W-Beijing genotype

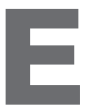
xtensively drug-resistant (XDR) tuberculosis (TB), defined as resistance to at least rifampicin and isoniazid (i.e. multidrugresistant (MDR)-TB) plus resistance to any fluoroquinolone and at least one of the three injectable anti-TB drugs (capreomycin, kanamycin or amikacin) [1], has recently emerged as a global health threat. According to the Global Anti-Tuberculosis Drug Resistance in the World, Report No. 4, published by the World Health Organization (WHO) [2], XDR-TB has been recorded in 45 countries and WHO estimates that $\sim 40,000$ cases of XDR-TB emerge worldwide every year. Not only are the highest XDR-TB rates in the countries of the former Soviet Union (FSU) and China, but XDR-TB has also emerged in countries where TB control has functioned effectively for many years $[3,4]$.

As attested by previous studies, MDR-TB emerged as a hazard to TB control worldwide in the 1990s. Treatment requires the use of second-line drugs, which are less effective, more toxic and costlier than the first-line drugs-based regimens [5]. As a consequence, treatment success rates are substantially lower than those of drug-sensitive TB cases [6]. Since 2006, even worse treatment outcomes and higher death rates have been demonstrated in XDR-TB [7, 8], especially in combination with HIV infection [9]. According to more recent studies, the treatment success of XDR-TB in low HIV prevalence countries ranges from $<40 \%$ in a joint report from Estonia, Germany, Italy and the Russian Federation [10], to $>60 \%$ in Peru [11]

Estonia, a republic of the FSU with a population of 1.34 million in 2005, was identified in 2000 as one of the MDR-TB "hot spots" and has consistently had one of the highest proportions of MDR-TB and XDR-TB in the world [2, 12].

After collapse of the Soviet Union, Estonia experienced substantial political, economic and social changes associated with declines in health

\section{AFFILIATIONS}

Dept of Pulmonary Medicine, University of Tartu, Tartu, Estonia.

CORRESPONDENCE

K. Kliiman

Dept of Pulmonary Medicine University of Tartu

Riia 167

51014 Tartu

Estonia

Fax: 3727318943

E-mail: kai.kliiman@kliinikum.ee

Received:

October 142008

Accepted after revision:

December 232008

STATEMENT OF INTEREST None declared. 
and a resurgence of TB. Following this, Estonia has implemented the WHO recommended Directly Observed Treatment, Short-Course (DOTS) strategy since 2000; in August 2001, Estonia started the DOTS-Plus project for treatment of MDRTB patients. As a result of the efficient work of the Tuberculosis Control Programme, the TB notification rate in Estonia decreased from 55.0 (in 2000) to 37.2 (in 2005) TB cases per 100,000 population [13], but the proportions of MDR-TB and XDR-TB have remained high. In 2005, MDR-TB accounted for $14.1 \%$ of new and $48.1 \%$ of previously treated cases; $20.6 \%$ of all MDR-TB cases were XDR-TB (11.9\% of new and $34.6 \%$ of previously treated TB cases, respectively) [13]. Strains of the W-Beijing genotype, known to associate internationally with large outbreaks of TB and increased virulence, are predominantly related to MDR-TB in Estonia [14] and have substantially contributed to the emergence of drug-resistant TB all over the country.

HIV prevalence in Estonia is rising. In 1999, only 12 new cases were diagnosed in Estonia and the overall number of HIVpositive cases was 64. However, during 2007, 633 new cases were detected and the total number of HIV-positive people reached 6,364 by the end of the year (Estonian Health Protection Inspectorate). In 2005, the estimated adult national HIV prevalence was $1.3 \%(0.6-4.3 \%)$ [15] and of all TB-cases, $8.4 \%$ were HIV-infected [13].

The low treatment success of MDR-TB patients in Estonia (53.2\% in 2005) [13] is particularly alarming when compared to the same indicator among non-MDR-TB patients $(83.4 \%)$. This background prompted the present authors to thoroughly analyse the effectiveness (the proportion of patients with a successful outcome) and efficacy (the proportion of patients with a successful outcome excluding defaulters) of treatment of MDR-TB and XDR-TB. Moreover, as the issue is poorly studied worldwide, the present authors concentrated on analysis of the factors associated with poor treatment outcome in patients with pulmonary MDR-TB and XDR-TB.

\section{MATERIALS AND METHODS \\ Patients and study design}

All patients with culture-confirmed pulmonary TB who started TB treatment in Estonia from January 2003-December 2005 were included in a retrospective case-control study to determine the predictors of poor treatment outcome in patients with pulmonary MDR-TB and XDR-TB. All patients had signs and symptoms consistent with TB. Patients without a final outcome (transferred out or still on treatment) were excluded. The study protocol was approved by the Ethics Committee on Human Research at the University of Tartu (Tartu, Estonia).

\section{Laboratory tests}

All laboratory tests were performed according to WHO recommendations by quality-assured laboratories [16]. All cultures were carried out with conventional Löwenstein-Jensen solid media and in BACTEC broth media using a radiometric BACTEC 460 or a fluorometric BACTEC MGIT960 system (all Becton Dickinson, Sparks, MD, USA). Drug susceptibility testing (DST) was performed as an indirect test by the proportion method on rifampicin $\left(2.0 \mu \mathrm{g} \cdot \mathrm{mL}^{-1}\right)$, isoniazid $\left(0.2 \mu \mathrm{g} \cdot \mathrm{mL}^{-1}\right)$, streptomycin $\left(4.0 \mu \mathrm{g} \cdot \mathrm{mL}^{-1}\right)$, ethambutol $\left(5.0 \mu \mathrm{g} \cdot \mathrm{mL}^{-1}\right)$, pyrazinamide $\left(100.0 \mu \mathrm{g} \cdot \mathrm{mL}^{-1}\right)$, capreomycin $\left(5 \mu \mathrm{g} \cdot \mathrm{mL}^{-1}\right)$, amikacin $\left(2.0 \mu \mathrm{g} \cdot \mathrm{mL}^{-1}\right)$, kanamycin $\left(5.0 \mu \mathrm{g} \cdot \mathrm{mL}^{-1}\right)$, ethionamide $\left(5 \mu \mathrm{g} \cdot \mathrm{mL}^{-1}\right)$ and ofloxacin $\left(2.0 \mu \mathrm{g} \cdot \mathrm{mL}^{-1}\right)$. If resistance was identified to isoniazid or rifampicin, the respective isolate was tested against second-line drugs. Quality assurance for DST was carried out by the WHO's Supranational Reference Laboratory in Stockholm, Sweden. For genotyping of the isolates, an insertion sequence (IS)6110based restriction fragment length polymorphism technique was used. The strains were spoligotyped as described by KAMERBEEK et al. [17] using commercially available membranes (Isogen, Maarssen, The Netherlands) [17]. The genotype families were defined based on published spoligotype profiles [18]. Clusters were defined as groups of strains with 100\% identical IS6110 patterns.

\section{Treatment}

Regimens to treat MDR-TB and XDR-TB cases were individually tailored on the basis of DST results. Typically, the treatment regimen contained at least four oral drugs used daily for the full course of treatment and an injectable medication until the monthly Mycobacterium tuberculosis culture converted to negative. After culture conversion, the injectable medication was continued three to five times weekly for an additional 2-3 months and discontinued thereafter. Usually, initial treatment for MDR-TB patients was provided on an in-patient basis and after culture conversion, patients were followed up in outpatient care under direct observation. During the outpatient treatment, patients received nutritional support (mean value US\$2.5 per day) and transportation reimbursement for the clinic visits. Treatment continued for 12-18 months after $M$. tuberculosis culture conversion, which was regularly pursued by $M$. tuberculosis smears and cultures up to the end of treatment. Estonia had full access to all categories of second-line drugs during the study period.

\section{Data collection}

A special database for collecting information was developed. Two groups of variables were collected: 1) patients' HIV status, demographic and socioeconomic characteristics; and 2) TBrelated data. Demographic and socioeconomic characteristics included age, sex, place of birth (Estonia or other), living situation (permanent place of living, occasional place of living or homeless), marital status (married/living as married or single/ divorced/widowed), previous imprisonment, place of residence (urban or rural), education (basic $(<9 \mathrm{yrs})$, secondary $(10-12$ yrs) and university ( $\geqslant 15$ yrs $))$, unemployment, presence of health insurance and alcohol abuse. Alcohol abuse was defined as registered alcoholism or any mention of medically significant excessive alcohol use in the medical record.

TB-related data included previous TB treatment, known TB contact, acid-fast bacilli (AFB) smear result and presence of cavitations on the chest radiograph performed at the time of TB diagnosis, disease detection method (passive or active), belonging of $M$. tuberculosis to W-Beijing genotype family and resistance to all tested first- and second-line TB drugs.

Data were extracted from patients' medical charts, bacteriological laboratory reports and the Tuberculosis Registry database.

\section{Definitions}

Standard WHO definitions for patient categories, treatment outcomes and MDR-TB and XDR-TB were used [19]. 
All patients were classified into one of the following two categories: new patients (never treated for TB) and patients previously treated for tuberculosis.

Treatment outcome was regarded as successful for patients considered "cured" or "completed", whereas "death", "default" and "failure" were combined as poor outcome. Any patient who was transferred outside Estonia during the course of treatment was qualified as "transfer out" and was excluded from analysis according to the protocol.

Treatment effectiveness was determined as the proportion of all patients with a successful outcome. The clinical efficacy of the DOTS-Plus treatment programme was measured as the proportion of all patients with a successful outcome excluding defaulters.

\section{Statistical analysis}

Comparisons of demographic, socioeconomic and HIV status and TB-related characteristics, as well as treatment outcome parameters between patient subgroups were performed using the Chi-squared test for categorical variables and the MannWhitney U-test for continuous variables. To estimate the predictors of poor treatment outcome among either MDR-TB or XDR-TB patients, multivariate logistic regression analysis with Wald statistical criteria using the backward elimination method was performed covering the variables in the two models of predictors: 1) patients' HIV status, demographic and socioeconomic characteristics; and 2) TB-related data. For variables with missing information, statistical analysis was performed for cases with complete information. A p-value $<0.05$ was regarded as statistically significant.

\section{RESULTS}

\section{Study population}

From January 2003-December 2005, 1,163 patients with culture-confirmed pulmonary TB were diagnosed in Estonia. Out of these patients, 256 had MDR-TB and of them, 60 had XDR-TB. Out of all patients, 48 died and six defaulted before starting TB treatment, hence, 1,109 patients were included in the cohort under assessment. Two patients were transferred out thereafter and treatment outcomes were thus assessed for 872 non-MDR-TB and 235 MDR-TB patients. The proportion of XDR-TB among MDR-TB patients was 23.0\% (54 out of 235)

Patients' median age was 43.2 yrs (range 15-80 yrs), 44.5 yrs for males (range 22-79 yrs) and 38.2 yrs for females (range 15$80 \mathrm{yrs}$ ). Baseline clinical characteristics and TB-related data are detailed in table 1. At the start of treatment, 186 of all MDR-TB cases $(79.1 \%)$ had resistance to all first-line anti-TB drugs. Patients with MDR-TB had median resistance to 5.0 anti-TB drugs (range 2-10), whereas those with XDR-TB had median resistance to 7.0 drugs (range $5-10 ; \mathrm{p}<0.001$ versus MDR-TB patients; table 2).

\section{Treatment outcomes}

In the 235 patients with MDR-TB, the proportion of patients with successful treatment outcome was $60.4 \%$ and the clinical efficacy of the treatment was $72.8 \%$ (table 3 ). Compared with patients with previously treated $\mathrm{TB}$, among those not previously treated for TB there was a significantly higher proportion of successful treatment $(71.0 \%$ versus $47.1 \%$, odds ratio (OR) 2.75, 95\% confidence interval (CI) 1.60-4.72; $\mathrm{p}<0.001)$, lower proportion of treatment failures $(4.6 \%$ versus $15.4 \%$, OR 0.26, 95\% CI 0.10-0.70; $\mathrm{p}=0.005)$ and lower mortality (7.6\% versus $20.2 \%$, OR 0.33 , 95\% CI $0.15-0.73$; $\mathrm{p}=0.005$ ).

In XDR-TB patients, the proportions of successful treatment outcome and treatment clinical efficacy were $42.6 \%$ and $50.0 \%$, respectively. In new XDR-TB cases, the proportion of defaulters was nonsignificantly higher than in the previously treated cases $(21.1 \%$ versus $11.4 \%$; $=0.342)$, but mortality was slightly lower $(15.8 \%$ versus $28.6 \% ; p=0.704)$. Compared with patients with MDR-TB, those with XDR-TB expectedly had a significantly lower proportion of successful treatment outcome (OR $0.39,95 \%$ CI $0.21-0.72 ; p=0.002$ ) and significantly lower clinical efficacy of treatment (OR $0.25,95 \%$ CI $0.13-0.51$; p $<0.001$ ).

\section{Risk factors associated with poor treatment outcome in MDR-TB patients}

HIV infection increased the risk of poor treatment outcome 10fold (OR 10.16, 95\% CI 1.17-88.84; $\mathrm{p}=0.04$ ) and previous TB treatment increased the risk almost three-fold (OR 2.88, 95\% CI $1.50-5.52 ; \mathrm{p}=0.001$; table 4). Resistance to ofloxacin (OR 2.30, $95 \%$ CI 1.17-4.51; $\mathrm{p}=0.02$ ) and positive AFB smear (OR 2.09, $95 \%$ CI 1.04-4.20; $\mathrm{p}=0.04)$ at the start of anti-TB treatment were independent risk factors of poor treatment outcome in MDRTB. Alcohol abuse $(p=0.07)$ was close to be significantly associated with poor treatment outcome in MDR-TB.

\section{Risk factors associated with poor treatment outcome in XDR-TB patients}

Living in an urban area (OR 19.76, 95\% CI 1.98-197.01; $\mathrm{p}=0.01$ ) was associated with poor treatment outcome in XDR-TB (table 5). Also, patients with positive AFB smear result at the start of treatment had more likely poor treatment outcome (OR 3.64, 95\% CI 1.03-12.88; p=0.045). Place of birth outside Estonia was insignificantly associated with XDR-TB poor treatment outcome.

\section{DISCUSSION}

The present study, designed to identify critical predictors of poor treatment outcomes patients with MDR-TB and XDR-TB, highlights opportunities for improvement in treatment outcomes for highly drug-resistant TB. It was shown that $72.8 \%$ of adherent MDR-TB patients and only half of adherent XDR-TB patients achieved a positive treatment outcome in a country with high prevalence of drug resistance, even though treatment strategies were implemented according to WHOrecommended MDR-TB treatment guidelines [20]. As a result of implementing the DOTS and DOTS-Plus strategies in Estonia since 2001, the prevalence of MDR-TB and the proportion of XDR-TB decreased slightly, from 49 XDR-TB cases (17.4\%) out of 281 MDR-TB cases during 2001-2003 to 27 (14.7\%) out of 184 cases in 2005-2007 (Estonian TB Registry, unpublished data).

It is well known that previous TB is the strongest risk factor for being ill with MDR-TB and XDR-TB [21]. Therefore, special attention has to be paid to improving the treatment adherence of re-treatment cases. The present study confirms that previous anti-TB treatment significantly increases the risk of poor treatment outcome in MDR-TB. This is in line with the results from previous studies [22]. The high proportion of MDR-TB 


\begin{tabular}{|c|c|c|c|c|}
\hline TABLE 1 & \multicolumn{4}{|c|}{$\begin{array}{l}\text { Demographic and socioeconomic characteristics, HIV status and tuberculosis (TB)-related data of all patients starting } \\
\text { TB treatment with culture-confirmed pulmonary multidrug-resistant (MDR)-TB, extensively drug-resistant (XDR)-TB and } \\
\text { non-MDR-TB in Estonia from January } 2003 \text { to December } 2005\end{array}$} \\
\hline Subjects $n$ & & 235 & 54 & 872 \\
\hline \multicolumn{5}{|c|}{ Demographics } \\
\hline Age yrs & & $43.2(34.1-53.0)$ & $45.0(33.0-53.8)$ & $45.0(36.1-55.4)$ \\
\hline$\leqslant 24$ & & $15(6.4)$ & $2(3.7)$ & $51(5.8)$ \\
\hline $25-44$ & & $111(47.2)$ & $24(44.4)$ & 347 (39.8) \\
\hline $45-64$ & & $94(40.0)$ & $25(46.3)$ & $363(41.6)$ \\
\hline$\geqslant 65$ & & $15(6.4)$ & $3(5.6)$ & $111(12.7)$ \\
\hline Born in Est & & $189(80.4)$ & $40(74.1)$ & $689(79.0)$ \\
\hline Seconda & & $132(56.2)$ & $30(55.6)$ & $495(56.8)$ \\
\hline Basic & & $91(38.7)$ & $23(42.6)$ & $316(36.2)$ \\
\hline Unknowr & & $2(0.9)$ & $0(0)$ & $10(1.1)$ \\
\hline Living alon & & $129(54.9)$ & $33(61.1)$ & $485(55.6)$ \\
\hline Homelessr & & $17(7.2)$ & $8(14.8)$ & $68(7.8)$ \\
\hline Unemployr & & $87(37.0)$ & $18(33.3)$ & $352(40.4)$ \\
\hline Alcohol ab & & $120(51.1)$ & $29(53.7)$ & $320(36.7)$ \\
\hline Presence c & health insurance & $145(61.7)$ & $33(61.1)$ & $510(58.5)$ \\
\hline Previous in & risonment & $55(23.4)$ & $12(22.2)$ & $161(18.5)$ \\
\hline \multicolumn{5}{|c|}{ TB-related data and HIV status } \\
\hline Previous $\mathrm{T}$ & treatment & $104(44.3)$ & $35(64.8)$ & $108(12.4)$ \\
\hline
\end{tabular}

Data are presented as $n(\%)$ or median (interquartile range), unless otherwise stated. AFB: acid-fast bacilli. ${ }^{\#}$ : single/divorced/widowed; " ${ }^{\circ}$ at the start of TB treatment.

and XDR-TB patients with a history of previous TB suggests that high rates of drug resistance could most probably be regarded as a consequence of the failure of TB control efforts before 2000 due to inadequate case management, interruptions in drug supply and use of inadequate drug regimens, or treatment defaults within recent years.

The present results indicate that a positive AFB smear result at the start of treatment is a risk factor of poor treatment outcome in both MDR-TB and XDR-TB. Smear-positive patients often have more advanced disease and a longer delay before obtaining medical care. In previous studies, prolonged patient delay has been found to be related to alcohol abuse [23], which was only slightly associated with poor treatment outcome in the current study.

The present authors found that poor outcome of XDR-TB treatment is strongly associated with living in an urban area. One speculative explanation of this phenomenon is the huge amount of enticements in the urban environment that interfere with treatment adherence, despite medical care being more accessible. The reason the same association was not observed in MDR-TB is possibly that the treatment of XDR-TB is tougher for the patient, owing to the greater number of medicines taken, and more challenging because of the side-effects of the treatment. Adherence can therefore be more affected or "fragile" as a result. Rationally, the most important tool for improving the treatment adherence of patients living in urban area is improved patient education. Knowing that poor outcome of XDR-TB treatment is strongly associated with urban living, it is easier to concentrate patient education more particularly on this XDR-TB population.

In the present study, as in several previous ones [2, 9], an association of poor MDR-TB treatment outcome with HIV infection was found. Despite a relatively low HIV prevalence (only 3.8\% of MDR-TB patients were HIV infected) the risk of poor treatment outcome in that particular subpopulation was 10 times higher. The rising HIV prevalence is intimidating and attention to early diagnosis of drug-resistant TB and early aggressive MDR-TB treatment should hence be particularly focused on HIV/MDR-TB-co-infected patients. Although not 


\begin{tabular}{|c|c|c|c|c|}
\hline \multirow[t]{2}{*}{ TABLE 2} & \multicolumn{4}{|c|}{$\begin{array}{l}\text { Drug resistance at start of treatment of all } \\
\text { patients with culture-confirmed pulmonary multi- } \\
\text { drug-resistant (MDR)-tuberculosis (TB), } \\
\text { extensively drug-resistant (XDR)-TB and non- } \\
\text { MDR-TB starting TB treatment in Estonia from } \\
\text { January } 2003 \text { to December } 2005\end{array}$} \\
\hline & & MDR-TB & XDR-TB & Non-MDR-TB \\
\hline \multicolumn{2}{|l|}{ Subjects $n$} & 235 & 54 & 872 \\
\hline \multicolumn{5}{|c|}{ First-line drugs } \\
\hline \multicolumn{2}{|l|}{ Isoniazid } & $235(100.0)$ & $54(100.0)$ & $106(12.2)$ \\
\hline \multicolumn{2}{|l|}{ Rifampicin } & $235(100.0)$ & $54(100.0)$ & $2(0.2)$ \\
\hline \multicolumn{2}{|c|}{ Streptomycin } & $220(93.6)$ & 49 (90.7) & $141(16.2)$ \\
\hline \multicolumn{2}{|c|}{ Pyrazinamide } & $63(26.8)$ & $13(24.1)$ & $4(0.5)$ \\
\hline \multicolumn{2}{|l|}{ Ethambutol } & $215(91.5)$ & $53(98.1)$ & $19(2.2)$ \\
\hline \multicolumn{2}{|c|}{ To all first line drugs } & $186(79.1)$ & $46(85.2)$ & $0(0)$ \\
\hline \multicolumn{5}{|c|}{ Second-line drugs } \\
\hline \multicolumn{2}{|l|}{ Amikacin } & 32 (13.6) & $15(27.8)$ & $4(0.5)$ \\
\hline \multicolumn{2}{|c|}{ Capreomycin } & $34(14.5)$ & $11(20.4)$ & $4(0.5)$ \\
\hline \multicolumn{2}{|l|}{ Kanamycin } & $153(65.1)$ & $53(98.1)$ & $7(0.8)$ \\
\hline \multicolumn{2}{|l|}{ Ofloxacin } & 68 (28.9) & $54(100.0)$ & $4(0.5)$ \\
\hline \multicolumn{2}{|c|}{ Protionamide } & $64(27.2)$ & $20(37.0)$ & $23(2.6)$ \\
\hline
\end{tabular}

addressed in the present study, a combination of TB treatment and antiretroviral therapy has been shown to improve treatment results in co-infected patients [24], thus providing limited grounds for optimism for this vulnerable patient group.

Several studies emphasise the important role of resistance to ofloxacin in poor MDR-TB treatment outcome [22, 25]. In the present study, the risk of poor treatment outcome was more than twice as high among patients in whom $M$. tuberculosis had resistance to ofloxacin. This finding further emphasises the importance of ofloxacin in MDR-TB treatment regimens and highlights the need for preserving susceptibility to ofloxacin, as well as pointing out the clinical value of ofloxacin resistance in the definition of XDR-TB. Contrary to the results of previous studies $[10,26]$, the present authors did not prove an association between poor treatment outcome and either resistance to injectable second-line TB drugs or resistance to all first-line TB drugs.

In the current study population, $53.6 \%$ of MDR strains belonged to the $\mathrm{W}$-Beijing genotype, but presence of this particular M. tuberculosis genotype was not associated with poorer treatment outcome. This is contrary to a previous report by LAN et al. [27], who documented Beijing genotype as an independent risk factor for treatment failure in TB.

At least two factors limited achievement of better treatment results in highly drug-resistant TB patients in the current study. First, the overall drug resistance rate was high, as MDRTB patients had median resistance to 5.0 TB drugs, whereas XDR-TB patients had median resistance to 7.0 drugs. Based solely on DST results, it was often impossible to create a treatment regimen with at least four effective TB drugs. Secondly, the proportion of defaulters in Estonia was high,

TABLE 3 Treatment outcome of all patients with culture-confirmed pulmonary multidrug-resistant (MDR)-tuberculosis (TB), extensively drug-resistant (XDR)-TB and non-MDR-TB starting TB treatment in Estonia from January 2003 to December 2005

\begin{tabular}{|c|c|c|c|c|c|}
\hline Outcome & Cure & Completion & Death & Failure & Default \\
\hline \multicolumn{6}{|l|}{ MDR-TB $\#$} \\
\hline Previously treated for TB & $46(44.2)$ & $3(2.9)$ & $21(20.2)$ & $16(15.4)$ & $18(17.3)$ \\
\hline Total & $135(57.4)$ & $7(3.0)$ & $31(13.2)$ & $22(9.4)$ & $40(17.0)$ \\
\hline OR $(95 \% \mathrm{Cl})^{\bullet}$ & $0.37(0.22-0.64)$ & $0.94(0.21-4.31)$ & $3.06(1.37-6.84)$ & $3.79(1.43-10.06)$ & $1.04(0.52-2.06)$ \\
\hline Never treated for TB & $8(42.1)$ & $1(5.3)$ & $3(15.8)$ & $3(15.8)$ & $4(21.0)$ \\
\hline Previously treated for TB & $14(40.0)$ & 0 & $7(28.6)$ & $10(20.0)$ & $4(11.4)$ \\
\hline Total & $22(40.7)$ & $1(1.9)$ & $10(18.5)$ & $13(24.1)$ & $8(14.8)$ \\
\hline OR $(95 \% \mathrm{Cl})^{5}$ & $0.92(0.30-2.85)$ & $0.95(0.85-1.05)$ & $1.33(0.30-5.89)$ & $2.13(0.51-8.96)$ & $0.48(0.11-2.21)$ \\
\hline$p$-value $e^{\S}$ & 0.88 & 0.17 & 0.70 & 0.29 & 0.34 \\
\hline \multicolumn{6}{|l|}{ Non-MDR-TB ${ }^{f}$} \\
\hline OR $(95 \% \mathrm{Cl})^{\# \#}$ & $0.33(0.21-0.52)$ & $1.97(0.83-4.65)$ & $1.89(0.88-4.06)$ & $1.04(1.00-1.08)$ & $2.59(1.42-4.76)$ \\
\hline$p$-value $\# \#$ & 0.001 & 0.12 & 0.10 & 0.001 & 0.001 \\
\hline
\end{tabular}

Data are presented $n(\%)$, unless otherwise stated. OR: odds ratio; Cl: confidence interval. ${ }^{\#}: n=235$; ${ }^{\circ}$ : comparison between never treated versus previously treated MDR-TB patients; ${ }^{+}: n=54 ;^{5}$ : comparison between never treated versus previously treated XDR-TB patients; ${ }^{f}: n=872 ;{ }^{\# \#}$ : comparison between never treated versus previously treated non-MDR-TB patients. 
TABLE 4 Demographic, socioeconomic, HIV and tuberculosis (TB)-related risk factors associated with poor treatment outcome of multidrug-resistant (MDR) pulmonary TB of all MDR-TB patients starting TB treatment in Estonia from January 2003 to December 2005

\begin{tabular}{|c|c|c|c|c|}
\hline \multirow[t]{2}{*}{ Variable } & \multicolumn{2}{|c|}{ Univariate analysis } & \multicolumn{2}{|c|}{ Multivariate analysis } \\
\hline & AOR $(95 \% \mathrm{Cl})$ & p-value & AOR $(95 \% \mathrm{Cl})$ & p-value \\
\hline \multicolumn{5}{|l|}{ Sex } \\
\hline Male & $1.87(1.01-3.45)$ & 0.05 & & \\
\hline \multicolumn{5}{|l|}{ Age yrs } \\
\hline$\leqslant 24$ & $0.42(0.08-2.20)$ & 0.30 & $0.24(0.02-3.23)$ & 0.28 \\
\hline $25-44$ & $1.13(0.36-3.60)$ & 0.83 & $0.86(0.15-5.00)$ & 0.87 \\
\hline $45-64$ & $1.48(0.46-4.75)$ & 0.51 & $1.74(0.30-10.04)$ & 0.53 \\
\hline$\geqslant 65$ & 1.0 & & 1.0 & \\
\hline \multicolumn{5}{|l|}{ Place of birth } \\
\hline Other & $1.37(0.71-2.62)$ & 0.35 & & \\
\hline \multicolumn{5}{|l|}{ Education } \\
\hline Basic & $1202(<0.001->1000)$ & 0.54 & & \\
\hline Secondary & $818(<0.001->1000)$ & 0.56 & & \\
\hline University & 1.0 & & & \\
\hline \multicolumn{5}{|l|}{ Living alone } \\
\hline Yes & $1.00(0.59-1.69)$ & 0.99 & & \\
\hline No & 1.0 & & & \\
\hline \multicolumn{5}{|l|}{ Homelessness } \\
\hline Yes & $1.80(0.67-2.82)$ & 0.25 & & \\
\hline No & 1.0 & & & \\
\hline \multicolumn{5}{|c|}{ Unemployment } \\
\hline Yes & 1.0 & & & \\
\hline \multicolumn{5}{|c|}{ Previous imprisonment } \\
\hline Yes & $1.56(0.84-2.87)$ & 0.16 & & \\
\hline No & 1.0 & & & \\
\hline \multicolumn{5}{|c|}{ HIV-seropositivity } \\
\hline Yes & $3.32(0.81-13.67)$ & 0.10 & $10.16(1.17-88.84)$ & 0.04 \\
\hline No & 1.0 & & 1.0 & \\
\hline \multicolumn{5}{|c|}{ TB related risk factors } \\
\hline \multicolumn{5}{|c|}{ Previous TB treatment } \\
\hline Yes & $2.75(1.60-4.71)$ & $<0.001$ & $2.88(1.50-5.52)$ & 0.001 \\
\hline No & 1.0 & & 1.0 & \\
\hline \multicolumn{5}{|c|}{ Known TB contact } \\
\hline Yes & $0.88(0.37-2.10)$ & 0.77 & & \\
\hline \multicolumn{5}{|l|}{ No } \\
\hline \multicolumn{5}{|c|}{ Cavitation on chest radiograph ${ }^{\#}$} \\
\hline Yes & $1.71(0.92-3.17)$ & 0.09 & $1.75(0.79-3.87)$ & 0.17 \\
\hline No & 1.0 & & 1.0 & \\
\hline
\end{tabular}




\section{TABLE 4 Continued.}

\begin{tabular}{|c|c|c|c|c|}
\hline \multirow[t]{2}{*}{ Variable } & \multicolumn{2}{|c|}{ Univariate analysis } & \multicolumn{2}{|c|}{ Multivariate analysis } \\
\hline & AOR (95\% Cl) & $p$-value & AOR $(95 \% \mathrm{Cl})$ & $\mathrm{p}$-value \\
\hline Yes & $2.07(1.19-3.60)$ & 0.01 & $2.09(1.04-4.20)$ & 0.04 \\
\hline No & 1.0 & & 1.0 & \\
\hline \multicolumn{5}{|c|}{ Detected with symptoms } \\
\hline \multicolumn{5}{|c|}{ W-Beijing genotype } \\
\hline Yes & $0.71(0.42-1.19)$ & 0.19 & $0.59(0.31-1.10)$ & 0.09 \\
\hline No & 1.0 & & 1.0 & \\
\hline \multicolumn{5}{|c|}{ Resistance to all first-line drugs } \\
\hline Yes & $0.94(0.49-1.78)$ & 0.84 & & \\
\hline \multicolumn{5}{|c|}{ Resistance to kanamycin } \\
\hline Yes & $1.99(1.12-3.52)$ & 0.02 & & \\
\hline No & 1.0 & & & \\
\hline \multicolumn{5}{|c|}{ Resistance to amikacin } \\
\hline Yes & $1.90(0.90-4.03)$ & 0.09 & & \\
\hline No & 1.0 & & & \\
\hline \multicolumn{5}{|c|}{ Resistance to capreomycin } \\
\hline Yes & $1.08(0.51-2.26)$ & 0.84 & & \\
\hline No & 1.0 & & & \\
\hline
\end{tabular}

AOR: adjusted odds ratio; $\mathrm{Cl}$ : confidence interval; AFB: acid-fast bacilli. ${ }^{\#}$ : at the start of TB treatment.

TABLE 5 Demographic, socioeconomic, HIV and tuberculosis (TB)-related risk factors associated with poor treatment outcome of extensively drug-resistant (XDR) pulmonary TB of all XDR-TB patients starting TB treatment in Estonia from January 2003 to December 2005

\begin{tabular}{|c|c|c|c|c|}
\hline \multirow[t]{2}{*}{ Variable } & \multicolumn{2}{|c|}{ Univariate analysis } & \multicolumn{2}{|c|}{ Multivariate analysis } \\
\hline & AOR $(95 \% \mathrm{Cl})$ & p-value & AOR $(95 \% \mathrm{Cl})$ & p-value \\
\hline \multicolumn{5}{|c|}{$\begin{array}{l}\text { Demographic, socioeconomic and HIV- } \\
\text { related factors }\end{array}$} \\
\hline \multicolumn{5}{|l|}{ Sex } \\
\hline \multicolumn{5}{|l|}{ Age yrs } \\
\hline$\leqslant 24$ & $<0.001(<0.001->1000)$ & 0.81 & & \\
\hline $25-44$ & $<0.001(<0.001->1000)$ & 0.82 & & \\
\hline $45-64$ & $<0.001(<0.001->1000)$ & 0.82 & & \\
\hline$\geqslant 65$ & 1.0 & & & \\
\hline \multicolumn{5}{|l|}{ Place of birth } \\
\hline Rural & 1.0 & & 1.0 & \\
\hline \multicolumn{5}{|l|}{ Education } \\
\hline Basic & $538(<0.001->1000)$ & 0.78 & & \\
\hline Secondary & $850(<0.001->1000)$ & 0.76 & & \\
\hline
\end{tabular}




\section{TABLE 5 Continued.}

Variable

University

Living alone

Yes

No

Homelessness

Yes

No

Unemployment

Yes

No

Alcohol abuse

Yes

No

Presence of health insurance

No

Yes

Previous imprisonment

Yes

No

HIV-seropositivity

Yes

No

\section{TB-related risk factors}

Previous TB treatment

Yes

No

Known TB contact

Yes

No

Cavitation on chest radiograph ${ }^{\#}$

Yes

No

AFB smear-positive ${ }^{\#}$

Yes

No

Detected with symptoms

Yes

No

W-Beijing genotype

Yes

No

Resistance to all first-line drugs

Yes

No

Resistance to kanamycin

Yes

No

Resistance to amikacin

Yes

No

Resistance to capreomycin

Yes

No
Univariate analysis

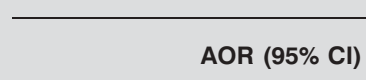

1.0

$0.53(0.17-1.65)$

1.0

$0.70(0.16-3.17)$

1.0

$0.89(0.26-2.80)$

1.0

$2.85(0.79-10.3)$

1.0

$1.02(0.34-3.08)$

1.0

$1.04(0.28-3.83)$

1.0

$1166(<0.001->1000)$

1.0

$1.35(0.44-4.16)$

1.0

$0.44(0.07-2.89)$

1.0

$2.28(0.62-8.40)$

1.0

$2.67(0.86-8.23)$

1.0

$1.02(0.30-3.47)$

1.0

$0.76(0.26-2.23)$

1.0

$0.40(0.07-2.18)$

1.0

$0.003(<0.001->1000)$

1.0

$1.16(0.35-3.89)$

1.0

$0.86(0.23-3.28)$

1.0
Multivariate analysis

AOR $(95 \% \mathrm{CI})$

p-value

0.28

0.65

0.85

0.98

0.96

0.79

0.60

0.39

0.22

0.09

$3.64(1.03-12.88)$

0.045

1.0

0.98

0.61

0.29

$0.20(0.02-1.90)$

0.16

1.0

AOR: adjusted odds ratio; $\mathrm{Cl}$ : confidence interval, AFB: acid fast bacilliz. ${ }^{*}$ : at the start of TB treatment. 
despite the provision of incentives and enablers, such as food and transport reimbursement, in the outpatient TB care setting, as well as intensive patient tracing. Out of all MDR-TB patients, $17.0 \%$ interrupted their treatment and the proportion of defaulters among XDR-TB patients was $14.8 \%$. Similar proportions of defaulters have been found in studies from Latvia [22] and South Africa [28]. In a report from Peru [29], the percentage of defaulters was lower, being $10.0 \%$ in MDR-TB patients and only $6.2 \%$ in $\mathrm{XDR}-\mathrm{TB}$, but according to a recent report on MDR-TB patients from South Korea, the proportion of defaulters was almost twice as high, reaching 32.2\% [30]. To improve treatment adherence and overall treatment success in highly drug-resistant $\mathrm{TB}$, it is therefore important to analyse the grounds for treatment default.

The strength of the present study is its capacity to provide representative country-wide data: all diagnosed cultureverified MDR-TB and XDR-TB patients who started TB treatment were included. This pertains also to laboratory data, since all XDR-TB-defining drugs were tested and DST results were quality-controlled. However, there were several limitations. Despite the inclusion of all diagnosed XDR-TB cases over a 3-yrs period, the absolute number of cases subjected to analysis was only 54 . Owing to the retrospective nature of the study, data on alcohol consumption and HIV status were missing in $12.3 \%$ and $8.5 \%$ of cases, respectively. Because of the unavailability of consistent representative data on drug abuse, this variable was excluded from the analyses.

The findings of the current study have several clear implications for TB control efforts. First, as the strongest risk factor of poor MDR-TB treatment outcome is HIV infection, extensive use of rapid diagnostic methods and immediate commencement of aggressive anti-TB treatment together with antiretroviral therapy is the way to improve treatment outcomes of HIV-infected TB patients. Secondly, the current results indicate that special attention should be paid to re-treatment cases in order to improve their adherence and treatment results. Thirdly, with better public information, communication and advocacy, it is possible to impel patients to seek medical care when they encounter their first TB symptoms and thereby to shorten patient delay and to detect less advanced disease.

It is known that interruption of the drug-resistant tuberculosis transmission cycle is possible if the cure rate is $>60 \%$. A cure rate of $\geqslant 80 \%$ is needed to achieve a 10 -fold reduction in multidrug-resistant tuberculosis incidence within 20 yrs [31]. In the light of the findings from the current study showing low treatment success rates in multidrug-resistant and extensively drug-resistant tuberculosis, it could be concluded that to reduce drug-resistant tuberculosis transmission in the community, improvement of treatment outcome, via ensuring adherence and paying special attention to HIV-infected tuberculosis patients, urban residents, re-treatment cases and those presenting with positive acid-fast bacilli smear, is especially needed in addition to extensive use of rapid diagnostic methods and highly effective aggressive tuberculosis treatment.

\section{ACKNOWLEDGEMENTS}

The authors thank A. Agejeva (Tartu University Hospital, Tartu, Estonia) for fingerprinting of the isolates, T. Kummik
(Tartu University Hospital) for her skilful work with drugsusceptibility testing and V. Hollo and P. Viiklepp (both Estonian Tuberculosis Registry, Tallinn, Estonia) for their professional help in data collection.

\section{REFERENCES}

1 Extensively drug-resistant tuberculosis (XDR-TB): recommendations for prevention and control. Wkly Epidemiol Rec 2006; 81: 430-432.

2 World Health Organization. Anti-tuberculosis drug resistance in the world. Report No.4. WHO/TB/2008.394. Geneva, World Health Organization, 2008.

3 Centers for Disease Control and Prevention (CDC). Emergence of Mycobacterium tuberculosis with extensive resistance to second-line drugs-worldwide, 2000-2004. MMWR Morb Mortal Wkly Rep 2006; 55: 301-305.

4 Shah NS, Wright A, Bai GH, et al. Worldwide emergence of extensively drug-resistant tuberculosis. Emerg Infect Dis 2007; 13: 380-387.

5 Gupta R, Kim JY, Espinal MA, et al. Public health. Responding to market failures in tuberculosis control. Science 2001; 293: 1049-1051.

6 Loddenkemper R, Sagebiel D, Brendel A. Strategies against multidrug-resistant tuberculosis. Eur Respir J 2002; 20: Suppl. 36, 66s-77s.

7 Migliori GB, Ortmann J, Girardi E, et al. Extensively drugresistant tuberculosis, Italy and Germany. Emerg Infect Dis 2007; 13: 780-782.

8 Kim HR, Hwang SS, Kim HJ, et al. Impact of extensive drug resistance on treatment outcomes in non-HIVinfected patients with multidrug-resistant tuberculosis. Clin Infect Dis 2007; 45: 1290-1295.

9 Gandhi NR, Moll A, Sturm AW, et al. Extensively drugresistant tuberculosis as a cause of death in patients coinfected with tuberculosis and HIV in a rural area of South Africa. Lancet 2006; 368: 1575-1580.

10 Migliori GB, Besozzi G, Girardi E, et al. Clinical and operational value of the extensively drug-resistant tuberculosis definition. Eur Respir J 2007; 30: 623-626.

11 Mitnick CD, Shin SS, Seung KJ, et al. Comprehensive treatment of extensively drug-resistant tuberculosis N Engl J Med 2008; 359: 563-574.

12 Becerra MC, Bayona J, Freeman J, Farmer PE, Kim JY. Redefining MDR-TB transmission "hot spots". Int J Tuberc Lung Dis 2000; 4: 387-394.

13 Hollo V. Tuberculosis Incidence in Estonia 2005. National Institute for Health Development, Tallinn, 2007.

14 Kruuner A, Hoffner SE, Sillastu H, et al. Spread of drugresistant pulmonary tuberculosis in Estonia. J Clin Microbiol 2001; 39: 3339-3345.

15 UNAIDS/WHO AIDS Epidemic Update: December 2006. http://data.unaids.org/pub/EpiReport/2006/2006_EpiUpdate_ en.pdf Date last accessed: October 14, 2008.

16 Laszlo A, Rahman M, Espinal M, Raviglione M. Quality assurance programme for drug susceptibility testing of Mycobacterium tuberculosis in the WHO/IUATLD Supranational Reference Laboratory Network: five rounds of proficiency testing, 1994-1998. Int J Tuberc Lung Dis 2002; 6: 748-756. 
17 Kamerbeek J, Schouls L, Kolk A, et al. Simultaneous detection and strain differentiation of Mycobacterium tuberculosis for diagnosis and epidemiology. J Clin Microbiol 1997; 35: 907-914.

18 Brudey K, Driscoll JR, Rigouts L, et al. Mycobacterium tuberculosis complex genetic diversity: mining the fourth international spoligotyping database (SpolDB4) for classification, population genetics and epidemiology. $B M C$ Microbiol 2006; 6: 23.

19 Laserson KF, Thorpe LE, Leimane V, et al. Speaking the same language: treatment outcome definitions for multidrug-resistant tuberculosis. Int $J$ Tuberc Lung Dis 2005; 9: 640-645.

20 World Health Organization. Guidelines for the Programmatic Management of Drug-resistant Tuberculosis.WHO/ HTM/TB/2006.361. Geneva, World Health Organization, 2006.

21 Faustini A, Hall AJ, Perucci CA. Risk factors for multidrug resistant tuberculosis in Europe: a systematic review. Thorax 2006; 61: 158-163.

22 Leimane V, Riekstina V, Holtz TH, et al. Clinical outcome of individualised treatment of multidrug-resistant tuberculosis in Latvia: a retrospective cohort study. Lancet 2005; 365: 318-326.

23 Kiwuwa MS, Charles K, Harriet MK. Patient and health service delay in pulmonary tuberculosis patients attending a referral hospital: a cross-sectional study. BMC Public Health 2005; 5: 122.
24 Dheda K, Lampe FC, Johnson MA, Lipman MC. Outcome of HIV-associated tuberculosis in the era of highly active antiretroviral therapy. J Infect Dis 2004; 190: 1670-1676.

25 Migliori GB, Lange C, Girardi E, et al. Fluoroquinolones: are they essential to treat multidrug-resistant tuberculosis? Eur Respir J 2008; 31: 904-905.

26 Migliori GB, Lange C, Centis R, et al. Resistance to secondline injectables and treatment outcomes in multidrug resistant and extensively drug-resistant tuberculosis cases. Eur Respir J 2008; 31: 1155-1159.

27 Lan NT, Lien HT, Tung LB, Borgdorff MW, Kremer K, van Soolingen D. Mycobacterium tuberculosis Beijing genotype and risk for treatment failure and relapse, Vietnam. Emerg Infect Dis 2003; 9: 1633-1635.

28 Holtz TH, Lancaster J, Laserson KF, Wells CD, Thorpe L, Weyer K. Risk factors associated with default from multidrug-resistant tuberculosis treatment, South Africa, 1999-2001. Int J Tuberc Lung Dis 2006; 10: 649-655.

29 Franke MF, Appleton SC, Bayona J, et al. Risk factors and mortality associated with default from multidrug-resistant tuberculosis treatment. Clin Infect Dis 2008; 46: 1844-1851.

30 Kim DH, Kim HJ, Park SK, et al. Treatment outcomes and long-term survival in patients with extensively drugresistant tuberculosis. Am J Respir Crit Care Med 2008; 178: 1075-1082.

31 Dye C, Williams BG. Criteria for the control of drugresistant tuberculosis. Proc Natl Acad Sci USA 2000; 97: 8180-8185. 